\title{
Utility of scatterplot patterns of automated hematology analysers in white blood cell disorders - A comparative study with peripheral blood smears
}

\author{
Tavish Gupta', Debdatta Basu² \\ ${ }^{1}$ MBBS (Intern), Jawaharlal Institute of Postgraduate Medical Education and Research, Puducherry, India, ${ }^{2}$ Professor \\ and Head, Department of Pathology, Jawaharlal Institute of Postgraduate Medical Education and Research, \\ Puducherry, India
}

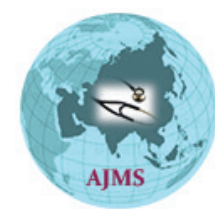

\section{A B S T R A C T}

Background: Advances in automated blood cell counting offer new possibilities to analyse leukocyte differentials by fluorescence RNA/DNA staining combined with scattered light intensity technologies. Aims and Objectives: The purpose of this study is to identify the scatterplot patterns in normal cell counts and various White Blood Cell (WBC) disorders and to assess their efficacy compared to a peripheral blood smear (PBS) in the diagnosis of WBC disorders. Materials and Methods: A three-phased descriptive study analysing a total of 933 EDTA blood samples. The total leucocyte count, differential leucocyte count, scatterplot patterns as obtained by automated cell counter and PBS were studied in 405 normal samples (Phase 1) and 415 samples with WBC abnormalities (Phase $2)$. In phase 3 , the scatterplots of 113 abnormal flagged samples were analysed and provisional diagnosis made in each case which was then compared with the diagnosis on the PBS. Sensitivity, Specificity, Positive and Negative predictive values (PPV \& NPV) were calculated. Results: A grid-based standard scatterplot of normal samples was generated as a reference plot in phase one. Based on the scatterplots obtained in known WBC abnormalities studied in phase two, the basic abnormal patterns specific to each of the individual abnormalities were discerned. The phase three study showed excellent correlation between scatterplots and PBS findings in neutrophilia, eosinophilia, lymphocytosis and leukopenia (sensitivity 92-100\%, specificity 98-100\%). Abnormalities suspected to be leukemia on the scatterplots had a relatively low sensitivity and PPV. Conclusion: WBC scatterplots can be used as a screening tool as it correlates well with various WBC disorders in the PBS.

Access this article online Website:

http://nepjol.info/index.php/AJMS DOI: 10.3126/ajms.v11i5.29049 E-ISSN: 2091-0576 P-ISSN: 2467-9100

Copyright (c) 2020 Asian Journal of Medical Sciences

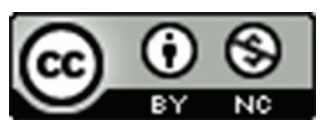

This work is licensed under a Creative Commons Attribution-NonCommercial 4.0 International License.

Key words: Automated hematology analyser; leukocyte abnormalities; peripheral blood smears; scatterplot pattern

\section{INTRODUCTION}

Diagnosis of various hematological disorders depend on blood counts, derived from automated cell counter and peripheral blood film examination. ${ }^{1}$ The automated hematology analyser has reduced the need for manual counts especially when many samples are analysed. The peripheral blood smear (PBS) examination is useful when a comprehensive morphological diagnosis is required but is time intensive and skill based. ${ }^{1,2}$

Recent advances in automated blood cell counting enable us to analyse leukocyte differentials by fluorescence RNA/DNA staining combined with scattered light 
intensity. ${ }^{1,3}$ It utilizes a combination of side scatter, forward scatter and fluorescence of the nucleic acid material to provide a concise and precise image of each white blood cell. The WBC -DIFF generated by most cell counters have good correlation with the manual reference method with a high sensitivity and shows excellent linearity in determining the White Blood Cell (WBC) differentials. ${ }^{4,5}$

Abnormal patterns in the WBC-DIFF and scatterplots generated by the system can provide a clue to various leucocyte disorders and has shown to be useful in the diagnosis of malaria and dengue and differentiate from the other causes of febrile illnesses. ${ }^{6,7}$ With this objective in mind, the present study was undertaken to identify the scatterplot patterns in normal WBC counts and in various WBC disorders (neutrophilia, eosinophilia, leukemia, reactive lymphocytosis and leukopenia) and to assess the efficacy of WBC scatterplot patterns compared to PBS in the diagnosis of WBC disorders

\section{MATERIALS AND METHODS}

A descriptive study was performed for a period of two months on EDTA blood samples studied within three hours of collection. Samples were analysed using the Sysmex XT-2000i hematology analyser (manufactured by Sysmex, Sysmex Sverige, MariosGata 13, S-434 37, Kungsbacka, Sweden). The parameters studied included total and differential leukocyte counts, scatterplot patterns and peripheral smears.

The study was conducted in three successive phases.

1. Phase One: Samples with normal total and differential counts as detected by the peripheral smear and the Sysmex Hematology Analyser were selected. The scatterplots of normal samples were studied and patterns among these were recognized.

2. Phase Two: Samples showing abnormal total and/ or differential leucocyte counts by peripheral smear findings were selected. The scatterplots of samples with abnormal WBC parameters (neutrophilia, eosinophilia, acute and chronic leukemia, reactive lymphocytosis, leukopenia, malaria) as seen in the PBS were analysed to detect for definitive patterns in them.

3. Phase Three: Consecutive samples flagged by the counter as abnormal for white blood cell parameters were selected. A provisional diagnosis was made on observing the scatterplots of samples (with the investigator being blinded to the smear findings) and the PBS of these samples were then studied to confirm the diagnosis.
Sample size (calculated using OpenEpi v3.01) - Phase One: The study was done to identify patterns which are seen in at least $25 \%$ of the normal reports. Using this percentage with $20 \%$ relative precision at $95 \%$ confidence limits the sample size calculated was 290 . However, phase 1 consisted of 405 samples. Phase Two: We assumed that a specified pattern in at least $60 \%$ of cases depicts abnormal cell counts and patterns. Using this percentage with 15\% relative precision at 95\% confidence limits the sample size required for this objective was calculated to be 114, although the study was done on 415 samples. Phase Three: 113 samples were studied considering the feasibility. Consecutive sampling was done.

\section{Statistical analysis}

- Phase One: The scatterplot patterns were analysed using the software program of the instrument.

- Phase Two: The frequency percentages of samples showing the identified patterns in each group of disorders - reactive lymphocytosis, leukopenia, leukemia, neutrophilia and eosinophilia are calculated. 95\% confidence intervals were also calculated.

- Phase Three: The diagnosis based on scatterplots are compared with the peripheral smear findings. Taking the peripheral smear findings as the gold standard, the sensitivity and specificity of the scatterplot patterns for various disorders among the samples collected was calculated.

\section{ETHICS}

Institution's ethical clearance was obtained prior to the start of the study. The study was done on EDTA blood samples submitted to the haematology lab of the institute for routine evaluation.

\section{RESULTS}

\section{Phase one}

The scatterplots of all the 405 cases with normal values as determined by the cell counter and PBS were analysed to find specific patterns within them.

After analysing the scatterplots of all the normal cases, 30 normal scatterplots were chosen which encompassed almost all the patterns found in the 405 scatterplots. Grids were superimposed on each scatterplot to get a better idea of the location of each cell region in the scatterplot. Further based on analysing the patterns of these 30 scatterplots, a prototype normal scatterplot was made manually.

Based on the normal scatterplots analysed and the prototype made, certain parameters were made for identifying a normal scatterplot such as the location of each cell region 
(based upon the grid which was superimposed), shape, size, density of the cells and their clustering, the angle each region makes with the horizontal and the dispersion of cells in the scatterplots (Figure 1).

1. Neutrophils are represented by cyan blue colour and consist of a single, thumb print shaped dense central area with peripheries showing a mild dispersion of cells. Some cells are present in the region joining the RBC debris and neutrophils while some more cells seen above the main neutrophil region most likely represent the band forms, precursor metamyelocytes and myelocytes (they tend to blend in with normal monocytes in terms of size and density).

2. Lymphocytes are represented by magenta colour and consist of a single spindle/tear drop shaped area consisting of a dense region in the bottom right of the spindle and the other regions showing dispersion of cells due to a wide variation in the size of the lymphocytes. These cells may merge with the monocytic region.

3. Monocytes are represented by green colour and consist of a single, quadrilateral shaped area with cells dispersed unequally and no consistent dense area. Variable densities of monocyte distributionis seen.

4. Eosinophils are represented by red colour and consist of a single, irregularly shaped area with a few cells dispersed unevenly and no consistent dense area. The eosinophilic area may be of reduced size in conditions where the eosinophil counts are near the lower border of their normal values.

5. $\mathbf{R B C}$ debris is seen as an irregular dark blue area present in the bottom right of the scatterplot called the Ghost region.

6. Basophils are appreciated in a different WBC-BASO scatterplot.

Phase two

A total of 415 samples in which the following abnormalities were detected by the PBS and flagged by the cell counter were selected.

- Neutrophilia - 196

- Eosinophilia - 83

- Lymphocytosis - 29

- Leukopenia - 78

- Leukemia - 29

i) Acute Myeloid Leukemia - 12

ii) Acute Lymphoblastic Leukemia - 8

iii) Chronic Myeloid Leukemia - 7

iv) Chronic Lymphocytic Leukemia - 2

Apart from these, five cases of Malaria and four cases of Dengue (diagnosed on serology), were also selected.

Neutrophilia - Increase in intensity of the neutrophil region was seen. Distortion of the shape, increased size, increased dispersion of cells to both sides of the neutrophil region (boomerang appearance), merging of cells with eosinophil region and an increase in the angle with the horizontal was seen. A significant proportion of the scatterplots showed cells above the neutrophil region resembling a vertical showering of cells upon the neutrophil region.

Eosinophilia - Increased intensity, increased size, elongation of shape, central clustering and an increased shift to the left was observed.

Lymphocytosis - Increase in the intensity of the cell clustering, widening or elongation of the region, reduced dispersion, increased clustering and merging with the monocyte region and a showering appearance of cells above the region was seen.

Leukopenia - Decrease in the intensity of all WBC regions, reduced clustering, increased dispersion, reduced size and absence of some cell regions (particularly eosinophils) was seen. There is either an increased number of neutrophils or lymphocytes relative to the normal percentage.

\section{Leukemia}

1) Acute Leukemia - Greying of cells with increased intensity and a meteor shape of cell clustering was present in most of the scatterplots and some showed a separate neutrophilic region whereas others showed a fusion of the lymphocytic and monocytic region. In Acute Myeloblastic leukemia, the grey area showed a bulge towards the right.

2) Chronic Leukemia - Most of the scatterplots showed greying of all areas with twin peaks, one in the lymphomonocytic region and the other in the neutrophilic region (twin mountain peak appearance). Variations such as a separate monocytic and eosinophilic regions, increased clustering and meteor shaped regions were seen.

\section{Additional cases}

1) Dengue: All cases showed lymphocytosis with increase in cell intensity and clustering along with a characteristic vertical showering of cells above the lymphocytic region. Increased dispersion of monocytes was also seen.

2) Malaria: Irregularity in the neutrophilic region with an increased density of cells towards the ghost region and variations such as greying of regions and a split in the neutrophilic region, pseudoeosinophilia was seen.

The scatterplots of neutrophilia, eosinophilia, lymphocytosis, leukopenia, leukemias, malaria and dengue are represented in Figure 1. 


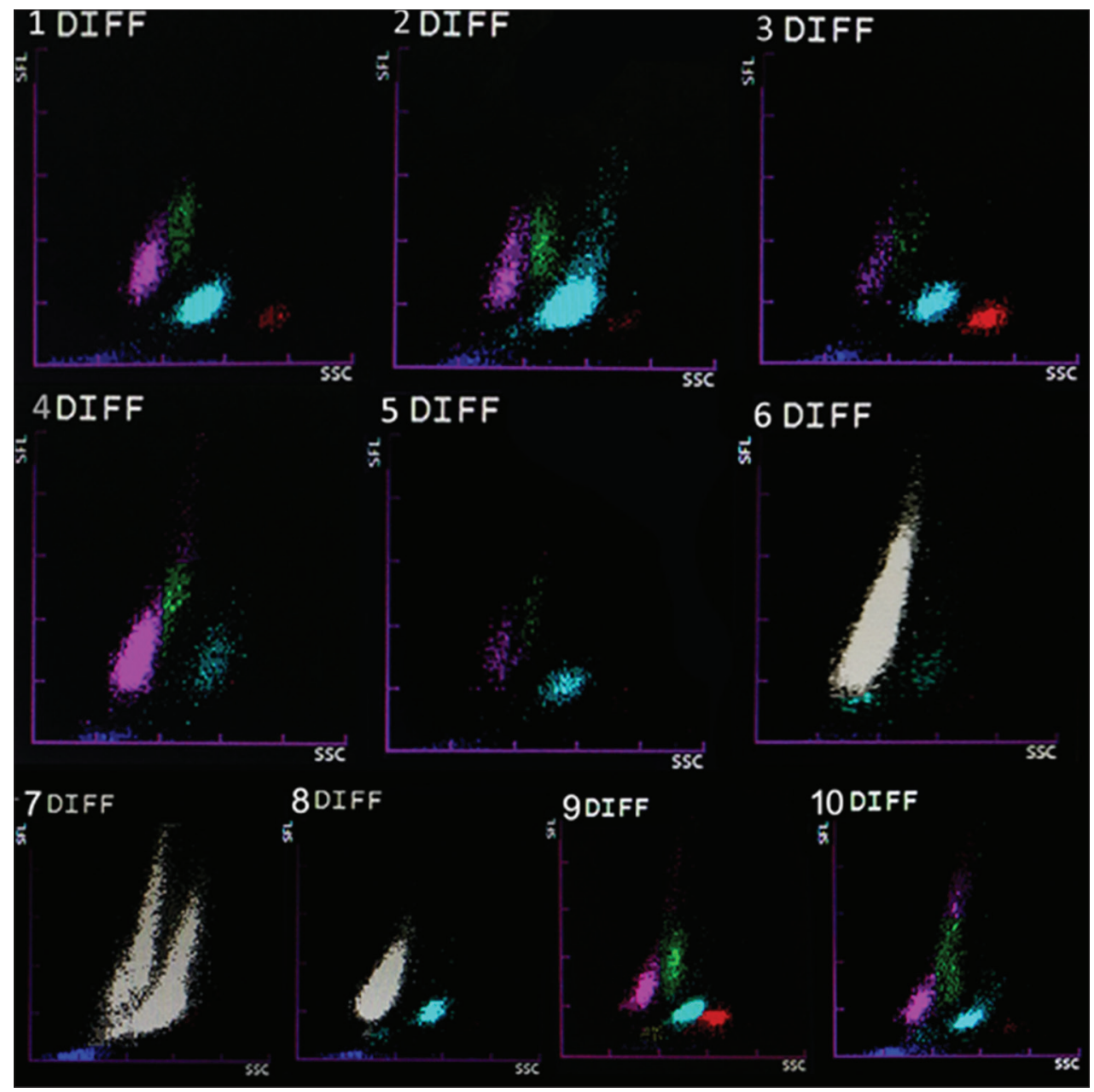

Figure 1: Scatterplots showing 1.) Normal Scatterplot 2.) Neutrophilia with boomerang appearance 3.) Eosinophilia 4.) Lymphocytosis 5.) Leukopenia with neutrophilic preponderance 6.) Acute Leukemia 7.) CML 8.) CLL 9.) Malaria 10.) Dengue

\section{Phase three}

A total of 113 samples were taken in which abnormalities were detected in the scatterplots and then compared with the peripheral smears to confirm the diagnosis.

- A presumptive diagnosis of neutrophilia was made in 39 cases on the scatterplots, all of which showed neutrophilic leucocytosis with left shift, band forms and toxic changes in the PBS

- Similarly, thirteen cases of lymphocytosis were detected on the scatterplots, all of which showed lymphocytosis in the PBS with reactive lymphocytes.

- Seventeen cases were considered as leucopenia on the scatterplots, all of which showed leukopenia under the PBS with either neutrophilic or lymphocytic preponderance.

- Of the fifteen cases with a presumptive diagnosis of eosinophilia on the scatterplots, fourteen showed eosinophilia on PBS

- Fourteen cases were considered as leukemia on the scatterplot. Three turned out to be neutrophilia with toxic changes with increased cell counts and merging of regions, while the rest were concordant with leukemia. There was considerable overlap among the scatterplot patterns of AML and ALL. All five cases of CML which were detected on the scatterplot were confirmed to be CML under the PBS. There were four cases, which were considered as monocytosis, nucleated red blood cells, leukopenia and neutrophilic left shift respectively because of splitting of regions, no greying; which reduced counts and left shift on the cell counter, turned out to be leukemia on the PBS. (One CML and three AML)

- Among the seven cases of monocytosis determined on scatterplot examination, six were confirmed under the peripheral blood smear examination whereas one case was determined to be AML-M2.

\section{Miscellaneous cases}

1. An abnormal scatterplot which showed two separate lymphocyte areas was unable to be determined by scatterplot analysis. On peripheral smear examination, there was presence of schizonts of Plasmodium Vivax 


\begin{tabular}{|c|c|c|c|}
\hline Abnormalities & $\begin{array}{l}\text { Scatterplot } \\
\text { Diagnosis }\end{array}$ & $\begin{array}{l}\text { Confirmed } \\
\text { on PBS }\end{array}$ & Discordant on PBS \\
\hline Neutrophilia & 39 & 42 & 3 cases were considered leukemia on scatter plot, turned out to be neutrophilia with toxic change \\
\hline Lymphocytosis & 13 & 13 & 0 \\
\hline Eosinophilia & 15 & 14 & 1 - Normal \\
\hline Leukemia & 14 & 15 & $\begin{array}{l}3 \text { - All three were neutrophilic leucocytosis with toxic change } \\
4 \text { cases were actually leukemia but diagnosed as leukopenia, NRBC's, Left shift and monocytosis }\end{array}$ \\
\hline Monocytosis & 7 & 6 & 1 - Degenerated cells \\
\hline NRBC'S & 7 & 1 & $\begin{array}{l}6 \text {-one was normal, two showed basophilia, one showed CML, one showed toxic change and } \\
\text { one showed monocytosis }\end{array}$ \\
\hline Miscellaneous & 1 & 1 & Schizonts of Vivax Malaria \\
\hline
\end{tabular}

\begin{tabular}{|c|c|c|c|c|}
\hline Abnormalities & Sensitivity & Specificity & $\begin{array}{l}\text { Positive } \\
\text { predictive } \\
\text { value }\end{array}$ & $\begin{array}{l}\text { Negative } \\
\text { predictive } \\
\text { value }\end{array}$ \\
\hline Neutrophilia & $92.86 \%$ & $100 \%$ & $100 \%$ & $95.5 \%$ \\
\hline Lymphocytosis & $100 \%$ & $100 \%$ & $100 \%$ & $100 \%$ \\
\hline Eosinophilia & $100 \%$ & $98.99 \%$ & $93.33 \%$ & $100 \%$ \\
\hline Leukopenia & $100 \%$ & $98.97 \%$ & $94.12 \%$ & $100 \%$ \\
\hline Leukemia & $73.33 \%$ & $96.94 \%$ & $78.57 \%$ & $95.96 \%$ \\
\hline Monocytosis & $100 \%$ & $99.07 \%$ & $85.71 \%$ & $100 \%$ \\
\hline NRBC'S & $100 \%$ & $94.64 \%$ & $14.29 \%$ & $100 \%$ \\
\hline
\end{tabular}

along with the ring forms. Thus, it was diagnosed to be malaria. In this case, the initial peripheral smear screen had missed the malarial parasite. The abnormal scatter plot made us relook and the schizonts were detected in the smear.

2. Seven scatterplots showing increased cells near the ghost region were presumed to be NRBC's but only 1 out of the 7 showed nucleated RBC's under the peripheral blood smear. The rest showed basophilia, monocytosis, CML and toxic change.

Table 1 gives a comprehensive review of all the cases in phase three, including the discordant cases.

Table 2 reflects the sensitivity and specificity of the scatterplot findings keeping the PBS findings as gold standard.

\section{DISCUSSION}

The study was conducted in three phases to find and assess patterns in scatterplots to aid in diagnosis of WBC abnormalities and a total of 933 samples were studied. It is the largest series comparing scatterplot patterns and peripheral smear findings in available literature.

In the first phase we were able to produce a grid based standard scatter plot of normal samples for reference based on superimposing 30 normal scatterplots encompassing the patterns found in all the 405 normal scatterplots. Such a descriptive finding has not been mentioned in literature, till date.

Most of the cells of a particular lineage occupy a specific area in the scatterplot, have a characteristic intensity \& clustering and each region makes a certain angle with the $\mathrm{x}$ axis depending upon the density, volume and nuclear complexity of the cells. Any deviation from these parameters (mentioned above in the results) usually tends to point towards some specific abnormalities.

During scatterplot analysis in the second phase; in cases of neutrophilia, lymphocytosis and eosinophilia; there was a general trend of increase in the cell intensity, clustering, angle made with the $\mathrm{x}$ axis and area occupied by the specific regions due to increase in the cell counts. In neutrophilia, there was increased number of cells above the neutrophilic region having increased size and nuclear complexity (determined to be immature granulocytes) and near the ghost region (older neutrophils undergoing degeneration) giving a boomerang appearance. This kind of an appearance has not been described in literature.

Lymphocytosis showed merging of the lymphocytes with the monocytic region representing the activated/reactive lymphocytes, with complex nuclear structure and increased cell size. This was characteristically seen in all our dengue cases and could provide a clue towards its diagnosis. ${ }^{6}$

Scatterplots of leukemia were distinctly abnormal with greying due to the drastically increased counts of blasts and immature cells and clustering, with a shift towards the neutrophil or lymphocyte region depending upon whether the leukemia was lymphoid or myeloid type. Once the basic abnormal scatterplot is detected it acted as a clue for PBS examination and other tests to diagnose leukemia., ${ }^{1,8}$

In the third phase neutrophilia, lymphocytosis, eosinophilia and leukopenia were detected with high 
accuracy (Refer Table 02). Excellent correlation has been reported amongst WBC parameters with respect to PBS findings as well as between different cell counter analysers. ${ }^{4,5}$

There was a discordance in a few cases as detailed in Table 01. Increase cellularity due to neutrophilic toxic change was incorrectly determined as leukemia three cases. The finding of prominent blue areas has been linked to presence of nRBC's and cellular debris ${ }^{4}$ However, six out of seven scatterplots showing prominent blue areas turned out to be CML with basophilia or toxic changes in neutrophils. In none of these smears, nRBC's were seen.

Cases of malaria showed abnormal clustering of cells around the ghost region. This finding was mentioned in previous studies detailing the usefulness of an automated cell counter in detecting malaria ${ }^{9,10}$. Pseudoeosinophilia has been mentioned as a very specific feature of malaria which we encountered in one out of the seven cases of malaria.

With the knowledge of the various scatterplot patterns obtained on automated analysers, paramedical staff, resident doctors, senior pathologists \& even treating physicians may be able to detect abnormalities especially in the absence of a clinical request for PBS examination and indicate ordering a PBS and examining it in detail. On the other hand, identifying a normal scatterplot may obviate the need for making PBS in each case, reducing the amount of resources required, cost and time. We recommend that attention be paid to the scatterplot patterns on cell counter in every case and based on that a decision may be made regarding evaluation of a PBS.

\section{ACKNOWLEDGEMENT}

This study was undertaken as a part of ICMR STS Project for Undergraduate Medical Students. (REFERENCE ID: 2017-02994)

\section{REFERENCES}

1. Sidra A, Ahmed I and Ali N. Utility of Peripheral Film Findings and Its Correlation with Automated Analyzer - An Audit from Tertiary Care Hospital. Journal of Laboratory Physicians 2017; 9.1:1-4. https://doi.org/10.4103/0974-2727.189233

2. Bain BJ. Diagnosis from the blood smear. N Engl J Med. 2005; 353:498-507.

https://doi.org/10.1056/NEJMra043442

3. Bruegel $M$, Nagel D, Funk M, Fuhrmann P, Zander $\mathrm{J}$ and Teupser D. Comparison of five automated hematology analyzers in a university hospital setting: Abbott Cell-Dyn Sapphire, Beckman Coulter DxH 800, Siemens Advia 2120i, Sysmex XE-5000, and Sysmex XN-2000 Clin Chem Lab Med. 2015; 53:1057-1071. https://doi.org/10.1515/cclm-2014-0945

4. Langford $\mathrm{K}$, Luchtman-Jones $\mathrm{L}$, Miller $\mathrm{R}$ and Walck $\mathrm{D}$. Performance Evaluation of the Sysmex XT2000i Automated Hematology Analyzer. Lab Hematol. 2003;9:29-37. PMID 12661825

5. Zimmermann M, Cremer M, Hoffmann C, Weimann K and Weimann A. Granularity Index of the Sysmex XE-5000 hematology analyzer as a replacement for manual microscopy of toxic granulation neutrophils in patients with inflammatory diseases. Clin Chem Lab Med 2011;49:1193-1198. https://doi.org/10.1515/CCLM.2011.188

6. Oehadian A, Michels M, Mast QDE, Prihanti D, Pushpita M, Hartantri $Y$, et al. New parameters available on Sysmex XE5000 hematology analyzers contribute to differentiating dengue from leptospirosis and enteric fever. Int. Jnl. Lab. Hem. 2015, $37,861-868$.

https://doi.org/10.1111/ijlh.12422

7. Mohapatra S, Samantaray JC, Arulselvi S, Panda J, Munot K and Saxena R. Automated detection of malaria with haematology analyzer Sysmex XE-2100. Indian J Med Sci 2011; 65:26-31. https://doi.org/10.4103/0019-5359.103163

8. Tefferi A, Hanson CA and Inwards DJ. How to Interpret and Pursue an Abnormal Complete Blood Cell Count in Adults. Mayo Clin Proc. 2005; 80:923-936. https://doi.org/10.4065/80.7.923

9. Jain M, Gupta S, Jain J and Grover RK. Usefulness of automated cell counter in detection of malaria in a cancer set up-Our experience. Indian J Pathol Microbiol 2012;55:467-473. https://doi.org/10.4103/0377-4929.107782

10. Sharma S, Sethi N, Pujani M, Kushwaha $S$ and Sehgal $S$. Abnormal WBC scattergram: a clue to the diagnosis of malaria. Hematology. 2013;18:101-105.

https://doi.org/10.1179/1607845412Y.0000000029

\footnotetext{
Authors Contribution:

TG - Collection of data, Interpretation of scatterplots and peripheral blood film, statistically analyzed and interpreted; reviewed the literature and prepared first draft of manuscript; DB - Concept and design of the study; interpreted the results, manuscript and critical revision of the manuscript; coordination of the overall study.

Work attributed to:

Department of Pathology, Jawaharlal Institute of Postgraduate Medical Education and Research, Puducherry, India.

Orcid ID:

Tavish Gupta - (1) https://orcid.org/0000-0002-5117-3003

Debdatta Basu - (1) https://orcid.org/0000-0001-5096-1406

Source of support: None, Conflicts of Interest: None
} 